\title{
Condição bucal, hábitos e necessidade de tratamento em idosos institucionalizados de Araras (SP, Brasil)
}

\author{
Oral condition, habits and treatment necessity \\ of institutionalized elders in Araras (SP, Brazil)
}

Michelle Cristina Lopes ${ }^{1}$

Viviane M aia Barreto de Oliveira ${ }^{2}$

Flávia M artão Flório ${ }^{3}$

\footnotetext{
${ }^{1}$ Universidade Estadual de Campinas. Rua das Primaveras 27, Jardim São Nicolau. 13602-024 Araras SP.lopesmic@pop.com.br ${ }^{2}$ Escola Bahiana de M edicina eSaúde Pública. ${ }^{3}$ Faculdade deO dontologia São Leopoldo M andic.
}

Abstract The aim of this study was to check the oral condition of the elderly citizens from the city of Araras (São Paulo, Brazil), and to evaluatetreatment needsfor this population. 118 volunteers were interviewed and 112 were examined, all of them with age above 60 years. The research was accomplished through a previous 14 question questionnaire that evaluated the patient general health, besides verified the self-knowledge of their oral health in addition to an intraoral physical exam, observing the oral conditions of the patients. In order to verify cavities prevalence, it was used an advocated criterion and index by WHO. It was observed that the systemic diseases that presented most prevalencewereinsomnia (40,67\%), followed by visual disturbances $(36,44 \%)$ and arthritis $(33,05 \%)$. In relation to the self-knowledge of the volunteers as to their oral condition, it was noticed that $90,67 \%$ of the individuals thought that oral condition does not affect their quality of life. With the intraoral exam, it was obtained a DM FT media equals to 30,6 with the lost component contributing to $93,9 \%$ of the cavities prevalence value $(p=28,7)$. This population requires special care focused on the oral health because besides having a high DMF-T, they also present inadequate oral health self-knowledge.

Key words Aged, Health of theelderly, O ral health
Resumo 0 objetivo deste trabalho foi verificar a condição bucal de idosos institucionalizados na cidade de Araras (SP) etambém avaliar a necessidadedetratamento dessa população. M etodologia: foram entrevistados 118 voluntários e examinados 112 idosos institucionalizados, acima de 60 anos, em Araras. A pesquisa foi realizada através de um questionário prévio com 14 questões para avaliar a saúde geral do paciente, além de verificação da autopercepção de saúde bucal e de exame físico intraoral, observando as condições bucais dos pacientes. Para conhecer a prevalência de cárie dentária, foram utilizados critérios e índices preconizados pela O rganização M undial da Saúde (OM S). Resultados: observou-sequeas doençassistêmicas que apresentaram maior prevalência foram a insônia (40,67\%), seguida de distúrbios visuais $(36,44 \%)$ e artrite $(33,05 \%)$. Em relação à autopercepção dos voluntários quanto à sua condição bucal, notou-se que 90,67\% dos indivíduos acham que a condição bucal não afeta a sua qualidade de vida. Com o exame intraoral, obteve-se um CPO-D médi o igual a 30,6 com o componente perdido contribuindo com $93,9 \%$ do valor da prevalência de cárie $(p=28,7)$. Conclusão: essa população precisa de cuidados especiais voltados à saúdebucal, poisalém depossuir um CPO-D alto apre senta autopercepção inadequada de saúde bucal. Palavras-chave I doso, Saúdedo idoso, Saúdebucal 
Introdução

A situação demográfica do país passou a sofrer mudanças intensas a partir da década de 1940, quando a população brasileira com idade superior a sessenta anos (idade na qual o adulto passa a ser considerado idoso) aumentou devido ao declínio de mortalidadee ao crescimento da expectativa de vida, alargando a base da pirâmide etária e diminuindo em direção ao topo. Subsequentemente, a queda da fecundidade na década de 1960 também contribuiu para o crescimento social ${ }^{1}$.

Assim, o envel hecimento da sociedade exige atualmente que o país se estruture para atender às necessidades dessa população crescente, pois entre os idosos são encontradas, com maior frequência, as doenças crônicas e as intervenções, apresentan do uma recuperação mais lenta, o que gera impacto significativo no setor de saúde pública². Além disso, ocorre desequilíbrio entre osteoblasto e osteoclasto, que resultam em osteoporose; o sistema imunológico torna-se menos potente, o uso de medicamentos leva à xerostomia, alteração de paladar, diminuição na coordenação motora, baixa autoestima e diversos fatores que contribuem para um aumento de doenças bucais, tornando-os indivíduos de alto risco para o aparecimento, principalmente, de cárie e doença periodontal ${ }^{3-5}$.

Para atender a essa nova demanda, a odontologia começa a investir em uma nova especialidade, odontogeriatria, oferecendo um tratamento diferenciado, uma vez que, nessa fase da vida, todos os sistemas orgânicos sofrem alterações, alertando o cirurgião-dentista quanto à inter-relação entre saúde geral e saúde bucal ${ }^{6,7}$, não esquecendo que o tratamento deveser individualizado, eliminando a abordagem de caráter universal ${ }^{8}$.

No intuito de disciplinar o atendimento ao idoso, Shay ${ }^{9}$ elaborou um guia para o profissional detectar fatores importantes antes do atendimento odontológico dos idosos, avaliado através do índice OSCARA (oral, sistêmico, capacidade, autonomia, realidade). Por meio desteíndice, seria possível avaliar a condição bucal dos pacientes, relacioná-la com a condição sistêmica, a capacidade de promover a própria higiene bucal e a realidade quecerca a saúde desse paciente.

Buscando compreender os aspectos psicossociais, a condição de saúde geral e bucal dessa população, algumas pesquisas começaram a ser desenvolvida nos últimos dez anos. Na pesquisa realizada por Anttila et al. ${ }^{10}$, fatores psicológicos, como a depressão, influenciam na saúde bucal dos idosos, elevando o risco de cárie edentulismo. Estudos realizados por Caldas Junior et al. ${ }^{11}$ concluíram que a prevalência de cárieéalta principalmente no grupo dos institucionalizados. Destaca-se também que a retração gengival afetando a raiz com lesões cariosas, nas pessoas acima de 75 anos, apresenta prevalência maior ao se comparar com a faixa de 50-75 anos.

Ettinger ${ }^{12}$ verificou que a cárie é o principal problema bucal nosidosos com 60 anos ou mais. Parajara \& Guzzo ${ }^{13}$ explicam que isso pode estar associado a fatores como redução do fluxo salivar pelo uso de medicamentos, dificuldade de higienização por problemas psicomotores ea alteração da dieta, que potencializam a ação da doença nessa população. Colussi e Freitas ${ }^{14}$ relatam que o CPOD foi menor em idosos pertencentes a grupos sociais da terceira idade, ao se comparar com idosos institucionalizados, enotase a falta de cirurgião- dentista para a realização de prevenções nessas instituições.

No Brasil, o apoio ao idoso é bastante precário ainda hoje, por se tratar de uma atividade estrita ao âmbito familiar. No entanto, a institucionalização do idoso tem ocorrido principalmente nos país desenvolvidos, em que asilos e casas derepouso tornam-se sofisticados em conforto eeficientes.

Associando as alterações dos arranjos familiares ao aumento da expectativa de vida, tem aumentado gradativamente a procura por instituições que atendam adequadamente a essa população. Entretanto, a maioria dos abrigos e asilos não está estruturada para suprir essa demanda.

Perante a nova realidade, esta pesquisa teve como objetivo verificar a condição bucal de idosos institucionalizados na cidade de Araras (SP) e também avaliar a necessidade de tratamento dessa população, para programações de ações futuras de intervenção.

\section{M ateriais e métodos}

Foram entrevistados 118 voluntários e examinados 112 idosos, acima de 60 anos, de ambos os sexos, residentes em cinco instituições de abrigo para idosos na cidade de Araras. Esses abrigos, um público, três filantrópicos e dois privados, representavam a totalidade de instituições da cidade (em uma das instituições têm-se duas categorias, publico e privado).

As pessoas acamadas, com problemas mentais ou dificuldade motora não foram descartadas da pesquisa, equando esses pacientes tinham dificuldades para responder ou não sabiam, as perguntas foram destinadas aos enfermeiros ou cuidadores que estavam dentro da instituição 
responsável pelo indivíduo. Apenas as questões relacionadas às condições gerais de saúde, como doenças sistêmicas e medicamentos, ou associadas ao tempo de estada nos asilos foram direcionadas a terceiros.

A pesquisa foi realizada através de um questionário prévio com 14 questões para avaliação da saúde geral do paciente, além de verificação da autopercepção de saúde bucal e de examefísico intraoral, observando as condições bucais dos pacientes.

O estudo foi conduzido após a aprovação do Comitê deÉtica ePesquisa do Centro Universitário Hermínio O metto-UNIARARAS eteveduração de seis meses. Todos os voluntários receberam e assinaram um Termo de Consentimento LivreeEsclarecido.

A avaliação clínica foi realizada por apenas um pesquisador, que passou por treinamento teórico-prático prévio objetivando assegurar a uniformidade de interpretação e critérios observados. Verificou-se nesta etapa que nove indivíduos haviam morrido, sendo quatro mulherese cinco homens; três indivíduos do sexo feminino saíram da instituição; oito pessoas novas entraram, perfazendo metade homens e metade muIheres; e dois idosos não permitiram a avaliação clínica, sendo um homem e uma mulher.

Para conhecer a prevalência de cárie dentária dos institucionalizados, foram utilizados crité rios e índices preconizados pela Organização Mundial da Saúde (OM S) ${ }^{15}$. A apuração dos resultados e a análise estatística dos dados foram realizadas através de análise exploratória.

\section{Resultados}

Foram aplicados formulários a 118 voluntários institucional izados, com idade média de 75,0 anos $( \pm 9,10)$, sendo 69 indivíduos do sexo feminino e 49 do sexo masculino. Esses indivíduos foram selecionados em cinco instituições decuidado ao idoso do município de Araras (SP) (Tabela 1).

Através do formulário, foram verificadas as seguintes variáveis: tempo de permanência na instituição, presença de problemas sistêmicos como diabetes, hipotensão, hipertensão, cardiopatias, mal deAlzheimer, mal de Parkinson, alergia a medicamentos ou cosméticos, labirintite, artrite reumatóide, distúrbios visuais, insônia, depressão. Também foram verificados 0 acesso ao tratamento médico e o uso de medicamentos.

Ao avaliar o tempo em que os idosos estão nas instituições, observou-se que 22,9\% ( $n=27)$ estão há menos deum ano, 45,8\% $(n=54)$ estão há me- nos de cinco anos, seguindo-se $30,5 \%(n=36)$ que estão há mais de cinco anos (Gráfico 1).

Os resultados obtidos a respeito de doenças sistêmicas foram distribuídos na forma de porcentagem, como pode ser verificado na Tabela 2. O bservou-se que a maioria dos idosos institucionalizados da amostra utilizam al gum tipo de medicação ( $82,20 \%, n=97)$ equeapenas $1,7 \%(n=2)$ destes o fazem sem acompanhamento médico.

As doenças sistêmicas que apresentaram maior prevalência foram a insônia $(40,67 \%$, $n=48)$, seguida de distúrbios visuais $(36,44 \%$, $n=43)$ e artrite $(33,05 \%, n=39)$.

Em relação à autopercepção dos voluntários quanto à sua condição bucal, notou-se que $90,67 \%$ ( $n=107$ ) dosindivíduosacham quea condição bucal não afeta a sua qualidade de vida (Gráfico 2).

A pós a aplicação dos formulários, foram realizados exames intrabucais. Obteve-se, após a coleta dos dados, um CPO-D médio igual a 30,6 $( \pm 3,2)$ com o componenteperdido contribuindo com $93,9 \%$ do valor da prevalência de cárie

Tabela 1. Distribuição de voluntários $(n=118)$ por sexo nas diferentes instituições.

\begin{tabular}{lccc}
\hline \multirow{2}{*}{$\begin{array}{c}\text { Contagem de sexo } \\
\text { Casa de idosos }\end{array}$} & \multicolumn{2}{c}{ Sexo } & \\
\cline { 2 - 3 } & $\mathbf{F}$ & $\mathbf{M}$ & Total global \\
\hline Santa Bárbara & 6 & 8 & 14 \\
N ossa Senhora do Patrocínio & 20 & 21 & 41 \\
Sayão Particular & 10 & 6 & 16 \\
Sayão SUS & 12 & 1 & 13 \\
São Judas Tadeu & 13 & 9 & 22 \\
Casa da Vovó & 8 & 4 & 12 \\
Total global & 69 & 49 & 118 \\
\hline
\end{tabular}

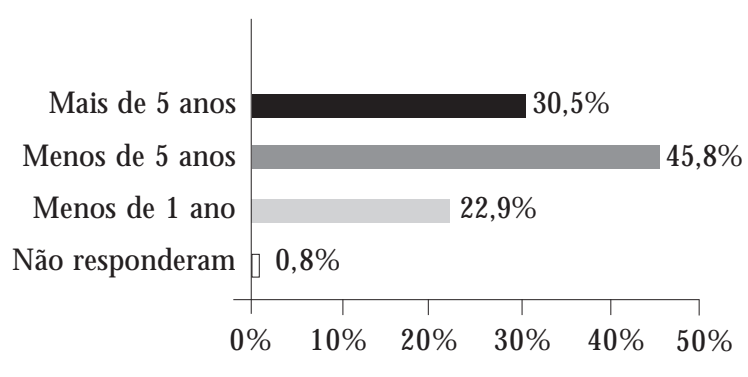

Gráfico 1. Tempo de permanência nas casas de repouso de idosos 
$(P=28,7)$. 0 Gráfico 3 mostra a média da distribuição dos componentes avaliados durante 0 exameintrabucal.

\section{Discussão}

Desde meados da década de 1970, osíndices epidemiológicos de doenças bucais mais prevalentes como a cárie começaram a apresentar me Ihoras para pessoas mais jovens, principalmente

Tabela 2. Características de uso de medicamentos, acometimentos sistêmicos e acesso a tratamento médico dos voluntários da amostra.

\begin{tabular}{lcc}
\hline \multicolumn{1}{c}{ Características } & $\begin{array}{c}\text { No de } \\
\text { pacientes (n) }\end{array}$ & $\begin{array}{c}\text { Porcentagens } \\
(\%)\end{array}$ \\
\hline Uso da amostra \\
Tratamenticação & 97 & 82,20 \\
Insônia & 95 & 80,51 \\
Distúrbios visuais & 48 & 40,67 \\
Artrite & 43 & 36,44 \\
Cardiopatia & 39 & 33,05 \\
Hipertensão & 28 & 23,72 \\
Alzheimer & 25 & 21,18 \\
Depressão & 13 & 17,79 \\
Labirintite & 12 & 11,01 \\
Diabete & 10 & 10,16 \\
Hipotensão & 5 & 8,47 \\
Mal de Parkinson & 5 & 4,23 \\
\hline
\end{tabular}

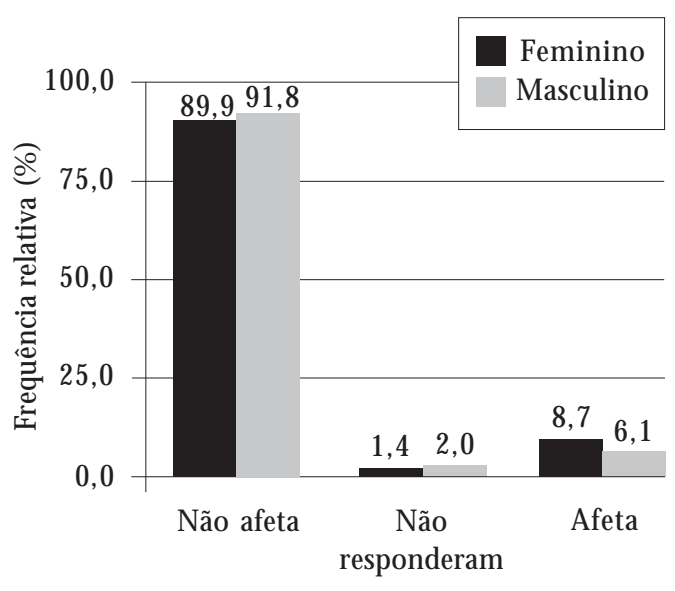

Condição bucal

Gráfico 2. Autopercepção da interferência da condição bucal na qualidade de vida. devido ao aumento da divulgação em massa de métodos preventivos ${ }^{16}$.

A odontologia vem evoluindo dia a dia; no entanto, à condição bucal eà necessidade detratamento da população muitas vezes não se dá a importância necessária. Diante disso, realizou-se um inquérito epidemiológico em idosos institucionalizados com 60 anos ou mais para se verificar a necessidade dessa população.

Segundo a Organização Mundial da Saúde (OM S), a meta preconizada para o ano 2000 era de que $50 \%$ da população com idade entre 65 e 74 anos tivesse vinte dentes ou mais na cavidade oral; e para 2010, de que apenas $5 \%$ fossem desdentados, o que foge da realidade atual ante os dados obtidos no presente trabalho e também em relação ao que afirmaram M elo et al. ${ }^{16}$, entre outros autores.

No Brasil, existem poucos estudos epidemiológicos nacionais. 0 primeiro deles foi realizado pelo M inistério da Saúdeem 1986. 0 segundo levantamento que incluiu pessoas com 65-74 anos foi o SB Brasil ${ }^{17}$, que é o mais recente e amplo levantamento. N este, avaliou-se um crescimento vertiginoso das sequelas de cárie dentária, em queo CPO-D médio obtido foi de 27,78, valor próximo ao encontrado nesta pesquisa $(30,6)$; dentre os componentes do CPO-D, o que prevaleceu foi o perdido, com 92,95\% ( $P=25,83)$, valor inferior ao de Araras $(93,9 \%, P=28,7)$. Em relação aos dentes hígidos, a média foi 3,40, valor superior aos idosos avaliados, em que se verificou o valor médio de $1,41( \pm 3,2)$.

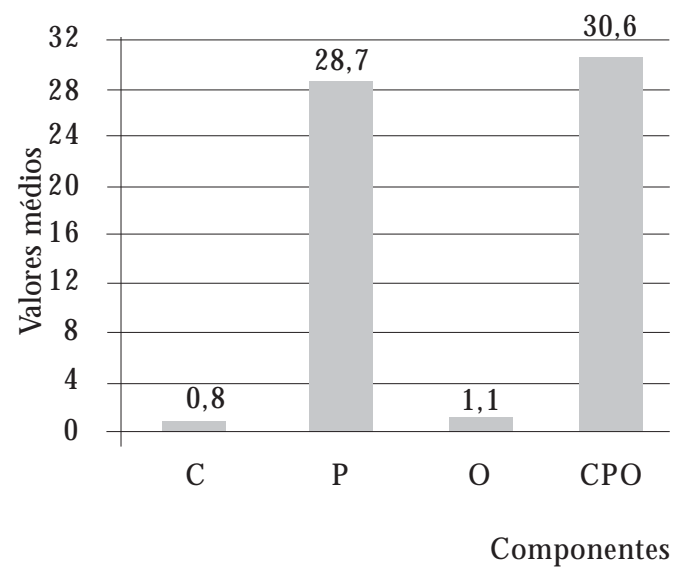

Gráfico 3. M édia do CPO-D, dentes hígidos e total de irrompidos obtida na avaliação clínica dos idosos. 
É possível que esses dados tenham sido diferentes da média pelo fato deserem apenas pessoas institucionalizadas, o que denota falta de tratamento adequado e acompanhamento odontológico, além da ausência de apoio familiar.

Esse grande número de dentes perdidos evidencia a falha ou a inexistência de tratamento restaurador ao alcance da população, que se vai acumulando até chegar a níveis extremos de extrações em idades avançadas ${ }^{18}$.

Notou-se, através deste estudo, que nesses idosos não foi possível atingir a meta da OMS, pois apenas $8,04 \%(n=9)$ da amostra submetida ao exameepidemiológico possuem vinte ou mais dentes, o que não correspondeà estimativa esperada para o ano 2000 , que era de $50 \%$.

À medida queos elementos dentários vão sendo perdidos, há perda considerável de eficiência mastigatória ${ }^{19}$, o que mais uma vez mostra a falta de autopercepção, em que $60,16 \%$ da amostra consideram a capacidade mastigatória boa, apesar do número elevado de dentes perdidos - números mais altos que os dados obtidos no SB Brasil, em que $44,58 \%$ dos pacientes acreditam possuir boa capacidade mastigatória ${ }^{17}$.

$\mathrm{Na}$ Austrália, apesar de $66 \%$ serem edêntulos, percebe-se o aumento do número de dentes presentes na cavidadeoral dosidosos, porém com cáries ${ }^{20}$.

Segundo estudo realizado por Fure ${ }^{21}$ na Sué cia, observou-se que, com o avanço da idade, a diminuição da saliva, o aumento do uso de medicamentos, do consumo de carboidratos e alterações da flora bucal são fatores que contribuem para frequência da lesão de cárie. No entanto, isso não se pode observar no presente estudo, pois a maioria dos idosos avaliados não possui dentes, portanto o índice de cárie foi baixo.

Cárie dentária, doença periodontal e outras doenças causadas pela presença e acúmulo de placa bacteriana e microorganismos na cavidade oral podem ser prevenidas através da eliminação e controle da placa com a execução de uma boa higienização, no entanto apenas $36,44 \%$ disseram realizá-la três vezes ao dia. Portanto, limitação física parcial ou total, dificuldades motoras, artrite, depressão, mal de Parkinson eAlzheimer são fatores que podem estar contribuindo para a não utilização de medidas de higiene oral .

Ao comparar dados obtidos por M elo et al. ${ }^{16} \mathrm{e}$ Shinkai e Cury', verificou-se que a limitação física $(17 \%)$ apresentada pelos autores teve resultados semel hantes com a dificuldade motora avaliada no presente trabalho (20,33\%). Em relação à depres- são, obteve-seum valor inferior ( $11,0 \%)$ ao secomparar com este estudo $(23,4 \%)$, e por fim avaliaram-se, além desses fatores, artrite (com 33,05\%), Alzheimer (17,8\%) e mal de Parkinson (4,2\%).

0 grande problema de ordem geral que afeta a saúde bucal são as doenças incapacitantes (mal de Parkinson avançado, distúrbios motores, artrite, Alzheimer e depressão). Assim, muitas vezes, énecessário reorientar o indivíduo com relação à higienização, se possível fazendo adaptações que facilitem a execução, como adaptações de cabos de escovas, uso de substâncias preventivas e terapêuticas ${ }^{8,16}$.

Outro aspecto importante a ser considerado é a capacitação dos cuidadores dos idosos em realizar a higiene bucal dos pacientes, garantindo uma higiene adequada àqueles que já não conseguem realizá-las adequadamente.

Assim, os cuidadores devem estar atentos a problemas bucais que podem interferir na saúde geral. É o caso das doenças periodontais, que elevam o risco de instalação e progressão das doenças cardiovasculares ${ }^{22}$. Assim também, problemas gerais podem interferir na condição bucal, como:

(1) Diabetes - aumenta a prevalência de candidíase, secura bucal inexplicável, lesões múltiplas de cárie e doença periodontal (não cedem a terapias convencionais) ${ }^{23}$.

(2) Alterações de pressão - comum em idosos, cujo tratamento farmacológico pode acarretar xerostomia, alteração do paladar e estomatite, o que requer cuidado 24 .

Portanto, novos estudos devem ser realizados em busca de dados que forneçam o grau de conhecimento dos cuidadores sobre a saúde bucal e possibilitem despertar o interesse em manter a saúde bucal.

\section{Conclusão}

A população avaliada precisa de cuidados especiais voltados à saúde bucal, pois além de possuir um CPO-D alto, apresenta autopercepção inadequada quanto à saúde bucal.

A informação e a orientação são importantíssimas na prevenção odontológica e devem ser extensivas a todas as equipes interdisciplinares.

A prática interdisciplinar é de extrema importância na odontologia geriátrica preventiva, através de consultas planejadas e periódicas de dentistas aos asilos, pois evita o contato tardio dos pacientes com profissionais da área odontológica. 


\section{Colaboradores}

M C Lopes trabalhou em toda a pesquisa, na avaliação dosinstitucionalizados, redação do artigo, interpretação dos dados e auxílio na confecção visual de tabelas e gráficos; VM B de Oliveira, no desenvolvimento da metodologia, revisão minuciosa do artigo, interpretação dos dados; FM Flório, na confecção da análise estatística, auxílio na confecção visual de tabelas e gráficos, interpretação de dados e revisão do artigo.

\section{Referências}

1. Pereira AC, Silva SRC, Watanabe MG, Meneghim MC, Queluz DP. Condições periodontais em idosos usuários do centro de saúde Geraldo de Paula Souza, São Paulo, Brasil. Rev Faculdade 0 dontol Lins 1996; 9(1):20-25.

2. Guedes JS. A saúde dos idosos no Estado de São Paulo. Informes em Saúde Pública 1, Secretaria de Estado de Saúde de São Paulo 1999; [s.d.]:13-21.

3. M ello ALSF, Padilha DM P. Instituições geriátricas e negligência odontológica. Rev Fac Odontol Porto Alegre 2000; 41(1):44-48.

4. Paunovich E, Saudowsky JM, Carter P. The most frequently prescribed medications in the elderly and their impact on dental treatment. Dent Clin N orth Am 1997; 41(4):699-726.

5. Duguid $Z A$, Singh $M, M$ artuscelli $G, M$ atthew $S$, Mallick S, Harrington DP, Papas AN, Papas AS. Prevalence of coronal and root caries in two high-risk groups. J Dent Res 2002; 81(n. special issue A):342.

6. Fajardo RS, Grecco P. O que o cirurgião-dentista precisa saber para compreender seu paciente geriátrico: parte 2. Aspectos fisiofuncionais. JBC Bras Clin Odontol Integr 2003; 7(41):432-438.

7. Oliveira JA, Ribeiro EDP, Bonachela WC, Capelozza ALA. Perfil do paciente odontogeriátrico da Faculdade de Odontologia de Bauru-USP. Rev Bras de Prótese Clín Lab 2002; 4(17):71-79.

8. Shinkai RSA, Cury AADB. O papel da odontologia na equipe interdisciplinar: contribuindo para a atenção integral ao idoso. Cad Saude Publica 2000; 16(4):1099-1109.

9. Shay K. Identifying the needs of the elderly dental patient: the geriatric dental assessment. Dent Clin North Am 1994; 38(3):449-523.

10. Anttila SS, Knuuttila MLE, Sakki TK. Relationship of depressive symptoms to edentulousness, dental health, and dental health behavior. Acta Odontotol Scand 2001; 59(6):406-412.

11. Caldas Junior $A F$, Figueiredo $A C L$, Soriano EP, Sousa EHA, M elo JBG, Vilela AS. Prevalência de cárie e edentulismo em idosos de Recife - Pernambuco Brasil. Rev Bras Ciênc Saúde 2002; 6(2):113-122.

12. Ettinger RL. Oral health needs of the elderly: an international review. Int Dent J 1993; 43(4):348-354.

\section{Agradecimentos}

Agradecemos a todos os voluntários que colaboraram com a pesquisa e às instituições que nos receberam com muito carinho.

13. Parajara F, Guzzo F. Sim, é possível envelhecer saudável! Rev Assoc Paul Cir Dent 2000; 54(2):91-99.

14. Colussi CF, Freitas SFT. Aspectos epidemiológicos da saúde bucal do idoso no Brasil. Cad Saude Publica 2002; 18(5):1313-1320.

15. Oliveira AGRC, Unfer B, Costa ICC, Arcieri RM. Levantamento epidemiológico básico de saúde bucal: manual de instrução. 4a ed. Genebra: Organização Mundial da Saúde; 1997.

16. M elo NSFO, Seto EPS, Germann ER. Medidas de higiene oral empregadas por pacientes da terceira idade. Pesq Bras Odontopediatria Clin Integr 2001; $1(3): 42-50$.

17. Brasil. Projeto SB Brasil 2003. Ministério da Saúde. Condição de saúde bucal da população Brasileira 20022003. Brasília: M inistério da Saúde; 2004.

18. Fernandes RAC, Silva SRC, Watanabe M GC, Pereira AC, M artildes MLR. U so e necessidade de prótese dentária em idosos que demandam um centro de saúde. Rev Bras de Odontol 1997; 54(2):107-110.

19. Jitomirski $F$, Jitomirski S. Odontogeriatria: a odontologia do futuro. Dens (Curitiba) 1987; 2(1):5-9.

20. Paley GA, Slack-Smith L, Grady MJ. Aged care staff perspectives on oral care for residents: Western Australia. Gerodontology 2004; 21(3):146-154.

21. Fure $\mathrm{S}$. Ten-year cross-sectional and incidence study of coronal and root caries and some related factors in elderly Swedish individuals. Gerodontology 2004; 21(3):130-140.

22. Nóbrega FJO, Garcia Filho OA, Seabra EG, Seabra FRG. Doença periodontal como fator de risco para o desenvolvimento de alterações cardiovasculares. Rev Bras Patologia Oral 2004; 3(1):41-47.

23. Brunetti RF, Montenegro FLB. Odontogeriatria: noções de interesse clínico. São Paulo: Artes Médicas; 2002.

24. M osegui GBG, Veras RP, Rozenfeld S, Vianna CM M . Avaliação da qualidade do uso de medicamentos por idosos. Rev Saude Publica 1999; 33(5):437-444.

Artigo apresentado em 13/12/2007

A provado em 27/06/2008

Versão final apresentada em 10/09/2008 